Esta obra forma parte del acervo de la Biblioteca Jurídica Virtual del Instituto unam de Investigaciones Jurídicas de la UNAM

www.juridicas.unam. $m x$

\title{
Acuerdos relevantes para la organización de los procesos electorales federal y estatales de 2012
}

A continuación se presenta una síntesis de los acuerdos tomados por el Consejo General del Instituto Federal Electoral y los correspondientes de los institutos electorales de Chiapas, Distrito Federal, Guerrero y Jalisco que destacan porque su contenido se refiere a cuestiones atípicas y novedosas dentro de la organización de sus respectivas elecciones. 
ACUERDO DEL CONSEJO GENERAL DEL INSTITUTO FEDERAL ELECTORAL POR EL QUE SE EMITEN LAS BASES Y LINEAMIENTOS O CRITERIOS ORIENTADORES PARA LA CELEBRACIÓN DE DEBATES ENTRE CANDIDATAS Y CANDIDATOS A CARGOS DE ELECCIÓN POPULAR EN EL PROCESO ELECTORAL FEDERAL 2011-2012. CG99/2012

Aprobado por votación unánime el 29 de febrero de 2012

\section{Acuerdo}

"Se aprueban las Bases y los Lineamientos o Criterios Orientadores para la celebración de debates entre candidatas y candidatos a cargos de elección popular en el Proceso Electoral Federal 2011-2012” (...)

"El Instituto Federal Electoral coordinará la realización de los dos debates entre las candidatas y los candidatos registrados a la Presidencia de la República, en los términos de la obligación que estatuye el artículo 70 del Código Federal de Instituciones y Procedimientos Electorales. A través de sus representantes, las candidatas y los candidatos comunicarán a la Presidencia del Consejo del Instituto y a la Comisión Temporal su decisión de participar en los debates.

Para el estudio y la definición de los debates a los que se refieren estas Bases, se establecerán un Comité Técnico y una Mesa de Representantes, a cuyas sesiones asistirán los consejeros electorales que integran la Comisión Temporal y el Secretario Técnico de ésta" (...)

"La producción de los debates estará a cargo de la Coordinación Nacional de Comunicación Social del Instituto Federal Electoral, de acuerdo con las instrucciones que reciba de la Comisión Temporal, a la que deberá informar sobre los trabajos realizados" (...)

"Los debates mencionados en el artículo 70 del Código Federal de Instituciones y Procedimientos Electorales serán transmitidos en vivo, ininterrumpidamente, por las estaciones de radio y canales de televisión de permisionarios públicos, incluyendo las de señal restringida" (...)

LINEAMIENTOS O CRITERIOS ORIENTADORES PARA LA CELEBRACIÓN DE DEBATES ENTRE CANDIDATAS Y CANDIDATOS A CARGOS DE ELECCIÓN POPULAR, DISTINTOS DE LOS 
PREVISTOS EN EL ARTÍCULO 70 DEL CÓDIGO FEDERAL DE INSTITUCIONES Y PROCEDIMIENTOS ELECTORALES

(...)

"El Instituto Federal Electoral reitera su disposición de apoyar, en los términos de la normativa aplicable y respetando la voluntad y las decisiones de los partidos y de las candidatas y candidatos, las iniciativas que éstos adopten libremente en materia de debates. Para ese fin promoverá la participación incluyente que favorezca la amplia expresión de las ideas y los proyectos" (...)

"Para atender a los principios de equidad y de trato igual, es pertinente que los organizadores de los debates convoquen oportunamente y por escrito a todas las candidatas y candidatos registrados conforme a lo dispuesto en el artículo 223 del Código Federal de Instituciones y Procedimientos Electorales, para la elección de que se trate, dejando constancia de la convocatoria formulada" (...)

DEBATES ENTRE CANDIDATAS Y CANDIDATOS A LA PRESIDENCIA DE LA REPÚBLICA ORGANIZADOS POR EL INSTITUTO FEDERAL ELECTORAL, DISTINTOS DE LOS PREVISTOS EN EL ARTÍCULO 70 DEL CÓDIGO FEDERAL DE INSTITUCIONES Y PROCEDIMIENTOS ELECTORALES

"Cuando medie acuerdo unánime y escrito de las candidatas y los candidatos a la Presidencia de la República y éstos expresamente soliciten al Instituto Federal Electoral que intervenga en la coordinación de los debates que aquéllos convengan, el Instituto Federal Electoral actuará, en lo conducente, conforme a lo establecido en las Bases relativas a los debates previstos en el artículo 70 del Código Federal de Instituciones y Procedimientos Electorales, así como en el Acuerdo que contenga el formato de dichos debates. La solicitud deberá presentarse ante la Presidencia del Consejo General del Instituto hasta quince días antes de la fecha o de las fechas establecidas por las propias candidatas y candidatos para la celebración del debate o los debates" (...)

DEBATES ENTRE CANDIDATAS Y CANDIDATOS REALIZADOS POR PERSONAS FÍSICAS Y MORALES SIN LA INTERVENCIÓN DEL INSTITUTO FEDERAL ELECTORAL 
"El Instituto Federal Electoral subraya que existe plena libertad para la organización de debates por parte de cualesquiera personas físicas o morales que deseen hacerlo, en relación con candidatas y candidatos a cargos de elección popular con motivo de las elecciones federales 2011-2012. Para ello, exhorta a los organizadores y comunicadores a observar las normas, principios y requerimientos del Proceso Electoral propios de una sociedad democrática, en el marco de la Constitución General de la República, los tratados internacionales y la legislación de la materia.

Estos debates -o programas que contengan debates como expresión de la libertad periodística- también podrán ser difundidos en la cobertura noticiosa de las campañas electorales, por cualquier medio de comunicación. Los que sean convocados o difundidos por estos medios, estarán sujetos a las disposiciones en materia de radio y televisión contenidas en el artículo 41 de la Constitución General de la República, el Código Federal de Instituciones y Procedimientos Electorales y el artículo 55 del Reglamento de Radio y Televisión en Materia Electoral" (...) 
ACUERDO DEL CONSEJO GENERAL DEL INSTITUTO FEDERAL ELECTORAL POR EL QUE SE EMITEN NORMAS REGLAMENTARIAS SOBRE ACTOS ANTICIPADOS DE CAMPAÑA DURANTE EL PROCESO ELECTORAL FEDERAL 2011 - 2012. CG92/2012

\section{Aprobado por votación unánime el 16 de Febrero de 2012 Acuerdo}

"Se aprueba el Acuerdo por el que se emiten normas derivadas del Código Federal de Instituciones y Procedimientos Electorales sobre actos anticipados de campaña durante el periodo que comprende del 16 de febrero al 29 de marzo de 2012 para el Proceso Electoral Federal 2011-2012, cuyo texto es el siguiente:

El periodo de "intercampaña" federal para el presente proceso electoral comprende del 16 de febrero al 29 de marzo de 2012. Durante el lapso que dura la "intercampaña" los partidos políticos no podrán exponer ante la ciudadanía por sí mismos ni a través de sus precandidatos y candidatos, sus plataformas electorales presentadas o registradas ante el Instituto Federal Electoral; ni los precandidatos y candidatos registrados ante sus institutos políticos podrán promoverse con el objeto de llamar al voto en actividades de proselitismo.

En el periodo de "intercampaña” no les está permitido a los partidos políticos, coaliciones, precandidatos y candidatos, el acceso a los tiempos del Estado en la radio y la televisión. Estos tiempos serán utilizados exclusivamente por las autoridades electorales. Durante la intercampaña, no podrán celebrarse debates entre los precandidatos, candidatos, partidos políticos y coaliciones. Quedan protegidas por la libertad periodística, las tertulias, los programas de opinión y las mesas de análisis político.

La libertad de expresión de los precandidatos y candidatos, así como el derecho de los medios de comunicación para ejercer su labor periodística al realizar entrevistas, están salvaguardados en todo momento. Los medios de comunicación podrán realizar entrevistas y difundir piezas noticiosas sobre los partidos políticos, coaliciones, precandidatos y candidatos postulados, con respeto absoluto a la equidad y a las disposiciones sobre la compra o adquisición de tiempo aire en la radio y la televisión.

En cualquier tiempo, los partidos podrán difundir propaganda política de carácter genérica conforme a los límites fijados en las leyes, 
-con excepción de la radio y a la televisión, toda vez que no tienen acceso a los tiempos del Estado en el periodo de "intercampaña"-; siempre y cuando no promueva candidaturas, ni solicite el voto a su favor para la Jornada Electoral Federal o incluya de manera expresa, mensajes alusivos al Proceso Electoral Federal.

Los actos anticipados de campaña a que se refiere el artículo 7, párrafo 2 del Reglamento de Quejas y Denuncias del Instituto Federal Electoral, quedan prohibidos a partir del 16 de febrero de 2012 y hasta la fecha de inicio de las campañas; por tanto, en dicho periodo queda prohibida también la promoción del voto a favor o en contra, la exposición de plataformas electorales, y emitir mensajes alusivos al Proceso Electoral Federal 2011-2012.

A más tardar el 1 de marzo de 2012 deberá quedar retirada toda propaganda de precampaña en bardas contratadas o asignadas por las autoridades, así como en espectaculares. Asimismo deberán retirarse las mantas colocadas en el equipamiento urbano y cualquier propaganda emitida por cualquier otro medio de difusión que haga referencia a precandidatos, precampañas o candidatos.

A partir del 16 de febrero de 2012 y hasta el 29 de marzo, podrá permanecer solamente la publicidad exterior sobre propaganda política genérica de los partidos políticos, siempre y cuando no haga referencia alguna a la promoción del voto a favor o en contra de partido, coalición o persona, a cargos de elección popular o al proceso electoral.

Las quejas o denuncias que sean presentadas con motivo del incumplimiento al presente Acuerdo serán resueltas mediante el procedimiento especial sancionador por el Consejo General o los consejos distritales de conformidad a las competencias establecidas para dichos órganos tanto en el Código Federal de Instituciones y Procedimientos Electorales, como en el Reglamento de Quejas y Denuncias.

Durante el periodo de intercampaña, los mensajes en radio y televisión que se difundan para dar a conocer informes de labores de funcionarios públicos a los que se refiere el artículo 228, numeral 5 del Código Federal de Instituciones y Procedimientos Electorales, no podrán hacer alusión a partidos políticos o incluir sus emblemas bajo ninguna modalidad" 
ACUERDO DEL CONSEJO GENERAL DEL INSTITUTO DE ELECCIONES Y PARTICIPACIÓN CIUDADANA, POR EL QUE SE DETERMINA LA MODALIDAD DE VOTO QUE GARANTICE EL EJERCICIO DEL DERECHO AL SUFRAGIO DE LOS CIUDADANOS CHIAPANECOS RESIDENTES EN EL EXTRANJERO EN EL PROCESO ELECTORAL LOCAL 2012

Aprobado por votación unánime el 13 de Marzo de 2012

\author{
Resultando
}

(...)

"La modalidad del voto electrónico es una vertiente que se encuentra en expansión en todo el mundo, países como Estonia, Francia, los Países Bajos, algunos estados en la Unión Americana, Canadá, España, Suiza y Austria, han recurrido a esta forma de votación por la cual el electorado puede sufragar por medio de un asistente digital como lo es la urna electrónica, o bien, a través de cualquier instrumento que cuente con conexión a Internet. El uso de las tecnologías de la información y de la comunicación en los quehaceres de la vida cotidiana cada vez en más frecuente en todo tipo de ámbitos, desde el escolar hasta el financiero, fomentando la creación de nuevas formas de organización social, que actúan en un entorno mundial de compresión espacio-tiempo en donde las barreras geográficas se han derrumbado, y en donde el uso de estas herramientas se vuelve cada vez más indispensable en la comunicación política. En esa tesitura, el transitar hacia el voto electrónico para el sufragio de los chiapanecos que residen en el extranjero resulta un ejercicio deseable por las ventajas que ofrece: El acercamiento de la prerrogativa a cualquier ciudadano sin importar el lugar en el que resida.

Asimismo, resulta factible desde territorio nacional mantener tanto la secrecía del voto, como el que éste se ejerza de forma universal, libre y directa, pues al contar con la tecnología adecuada en materia de seguridad se puede garantizar que el ciudadano ejerza su derecho bajo dichas previsiones, y adquirir la tecnología de seguridad adecuada se puede garantizar la certeza tanto para la emisión como para la recepción del voto. La seguridad de que al optar por esta modalidad, el control del proceso electivo, permanece dentro de territorio nacional, 
pues al adquirir tecnología de seguridad adecuada, se puede garantizar la certeza tanto para la emisión como para la recepción del voto. Cabe señalar, que sobre la introducción del voto electrónico existen pocos argumentos en contra, la mayoría de ellos centrados en preocupaciones sobre la seguridad de los resultados, así como en los costos de adquisición de tecnología, sin embargo éstos no han logrado socavar la principal cualidad de la transmisión de información por este medio: su impacto a nivel mundial. En efecto, entado el mundo existe una identificación con esta clase de tecnología, fomentada por el constante desarrollo de sistemas de seguridad, por la cual el uso de estos medios en la transmisión de datos no sólo no ha disminuido, sino que se ha incrementado. En virtud de ello, y con el objeto de acercar al electorado residente en el extranjero las opciones que les permitan ejercer su derecho al voto para la elección de la fórmulas de diputados especiales al Congreso del Estado de Chiapas en el proceso electoral local 2012, se analiza la posibilidad de implementar el voto electrónico, resultando una opción que no sólo garantizaría una mayor participación de la ciudadanía, sino que permitirá a este Instituto contar con información certera disminuyendo las posibilidades de error en el cómputo, al mismo tiempo que garantiza la libertad del sufragio" (...)

"Es por ello que haciendo uso de los beneficios de la tecnología, este Consejo General ha considerado que es viable la implementación del voto electrónico por internet para recabar el sufragio de los ciudadanos chiapanecos residentes en el extranjero, para elegir al la fórmula de Diputados especial al Congreso del Estado de Chiapas en el proceso electoral local ordinario 2012, dado que es una modalidad que se encuentra en expansión en todo el mundo. Aunado a lo anterior, es de considerar que este mecanismo provee diversos beneficios que revisten de razonabilidad su inversión, tales como que es ecológico, arroja resultados de manera inmediata, simplifica los procedimientos de votación, disminuye el error humano en el cómputo y reduce el número de funcionarios necesarios para implementación, así como para la capacitación en su desarrollo" (...)

\section{Acuerdo}

"Se aprueba como mecanismo para recabar el sufragio de los ciudadanos chiapanecos residentes en el extranjero para la elección de la fórmula de Diputados especial en el proceso electoral local 2012, la vía electrónica por internet” (...) 
ACUERDO DEL CONSEJO GENERAL DEL INSTITUTO ELECTORAL DEL DISTRITO FEDERAL POR EL QUE SE APRUEBAN LOS MECANISMOS PARA RECABAR EL VOTO DE LOS CIUDADANOS DEL DISTRITO FEDERAL RESIDENTES EN EL EXTRANJERO PARA ELEGIR AL JEFE DE GOBIERNO EN EL PROCESO ELECTORAL ORDINARIO 2011-2012. ACU-69-11

Aprobado por votación unánime el 31 de Octubre de 2011

\author{
Considerando
}

(...)

"La modalidad del voto electrónico por Internet, cumple con las características esenciales del voto. En primer término la universalidad del sufragio se garantiza para todos los ciudadanos que cumplan con los requisitos legales para ejercer su derecho al voto - contar con dieciocho años cumplidos y tener un modo honesto de vivir situación que se verifica cuando se integra el Listado Nominal; anteriormente este derecho se encontraba limitado por una razón extralegal, consistente en encontrarse fuera del territorio nacional y ahora se rompe con esa barrera, por primera ocasión, a nivel local; es libre porque se ejerce con absoluta libertad y responsabilidad, en este caso; desde el hogar, oficina o lugar que el ciudadano haya destinado para ejercer su derecho y siempre que se haya inscrito en el Listado Nominal correspondiente, únicamente necesita un medio electrónico o computadora conectada a Internet desde el extranjero; es directo porque únicamente el propio ciudadano puede entrar al sistema y solicitar su contraseña. La secrecía se manifiesta en dos principales etapas, la primera con el hecho de solicitar la contraseña que es única y que se asigna de manera aleatoria, sin conocimiento de ningún funcionario público sino a través de un sistema con el cifrado que no permite identificar al ciudadano con ésta y en segundo término, cuando el ciudadano realiza la votación; el voto se transporta por la red a través de un algoritmo cifrado con altos niveles de seguridad que no permite identificar los datos personales del ciudadano con su elección y tampoco el sentido de su voto.

Debe resaltarse que tanto el voto postal como el voto electrónico por internet, se adoptan como mecanismos para que el elector re- 
sidente en el extranjero tenga alternativas que le faciliten sufragar de manera responsable; en el entendido que cualquiera de esas opciones se sujetará a procedimientos aprobados por esta autoridad, tendentes a garantizar las características de libertad, intransferibilidad y secrecía propias de esta prerrogativa ciudadana. Con ello, esta autoridad mantiene el control y seguridad del proceso electoral en su conjunto" (...)

\section{Acuerdo}

"Se aprueban como mecanismos para recabar el sufragio de los ciudadanos del Distrito Federal residentes en el extranjero para la elección de Jefe de Gobierno en el proceso electoral ordinario 20112012, las vías postal y electrónica por Internet” (...)

MEDIANTE EL QUE SE APRUEBA EL TIPO, INTEGRACIÓN Y UBICACIÓN DE LAS CASILLAS ELECTORALES A INSTALAR EN ELECCIONES COINCIDENTES, A EFECTO DE CONVENIR CON EL INSTITUTO FEDERAL ELECTORAL EN LAS DIVERSAS MATERIAS RELACIONADAS CON EL PROCESO ELECTORAL A CELEBRARSE EN EL AÑO 2012. APROBACIÓN EN SU CASO. 049/ SO/15-11-2011

Aprobado por votación unánime el 15 de Noviembre de 2011

Considerando

“Tomando en cuenta que en el año 2012 se realizará la jornada electoral de la elección local, el mismo día en que se llevará a cabo la de la elección federal, por lo que en el marco del análisis del Convenio a suscribir con el Instituto Federal Electoral; los integrantes del Consejo General y el personal técnico operativo de este instituto, a través de diversas reuniones se han enfocado al estudio de las actividades que se deberán desarrollar de forma concurrente con las del Instituto Federal Electoral, respecto a los trabajos concernientes a los procedimientos de ubicación, integración y funcionamiento de las mesas directivas de casilla a instalar el día de la elección, con la finalidad de que los trabajos que a cada órgano electoral corresponde, se realicen de forma satisfactoria a las exigencias que sus respectivas legislaciones electo- 
rales requieren y, sobre todo, se garantice la efectividad del sufragio de manera transparente, ordenada y asequible a los ciudadanos" (...)

"De conformidad con la información recabada en los encuentros nacionales de órganos electorales del País en los que este instituto ha participado y en las visitas realizadas a diversos órganos electorales, en los cuales, se ha observado la posibilidad de instalar tres tipos de casillas a saber: Única, Mixta o ampliada y Espejo o Separada. La primera se refiere a que su integración será la que señala la ley de la materia con cuatro propietarios y tres suplentes generales, quienes se encargarán de recibir la votación, tanto de las elecciones federales como las del Estado. Asimismo, realizarán todas las actividades que conciernen a ambas elecciones como son: instalación, conteo de boletas, llenado de actas, recepción de la votación y de escritos de protesta o de incidentes, escrutinio y cómputo de cada una de las elecciones federales y locales, así como la entrega de los paquetes al órgano electoral correspondiente.

Por su parte, la casilla mixta o ampliada, se integra con un Secretario más (aparte de los cuatro propietarios), quien se encargará de las funciones concernientes a la elección local. Por último, la casilla separada o espejo, consiste en la instalación de dos casillas: una para cada elección (federal y local) conforme a las reglas y procedimientos establecidos en las legislaciones respectivas.” (...)

"Se advirtió que la casilla separada o espejo, representa un dinamismo natural propio que las legislaciones respectivas les confieren a cada órgano electoral, sin embargo, ante la eventual circunstancia de realizar elecciones coincidentes, se hace imperativo señalar a detalle cada uno de los actos de integración de las casillas, desde la insaculación de los ciudadanos hasta su total instalación el día de la jornada electoral, como es el caso del sorteo del mes, el cual, no debe coincidir con el de la elección federal a fin de que no se insacule por ambos institutos a las mismas personas; la igualdad de retribuciones de los capacitadores y funcionarios de casilla, incluyendo la carga laboral, a fin de que cuenten con los mismos beneficios de ambos organismos electorales; así como la instalación de ambas casillas, el orden para votar y las medidas de seguridad que se deben de observar para la emisión libre, secreta y directa del voto, con la finalidad de que la votación fluya de forma ordenada, con el mayor ritmo posible, y así, evitar contratiempos." (...) 


\section{Acuerdo}

"Se determina que para el día de la elección coincidente a efectuarse el primer domingo de julio del año 2012, deberá ser la casilla separada y/o espejo, para tal efecto el Instituto Electoral del Estado de Guerrero deberá proveer las acciones conducentes para el efecto de integrar e instalar las casillas que se encargarán de recibir la votación de las elecciones locales, en los plazos y términos establecidos en la legislación electoral del Estado" (...)

"El Consejo General del Instituto Electoral del Estado de Guerrero, aprueba la instalación de la casilla única en los lugares y por los motivos específicos que al respecto se señalen" (...) 
ACUERDO DEL CONSEJO GENERAL DEL INSTITUTO ELECTORAL Y DE PARTICIPACIÓN CIUDADANA DEL ESTADO DE JALISCO MEDIANTE EL CUAL APRUEBA LOS LINEAMIENTOS PARA EL USO DEL SISTEMA ELECTRÓNICO PARA LA RECEPCIÓN DEL VOTO MEDIANTE URNA ELECTRÓNICA, EN EL PROCESO ELECTORAL ORDINARIO 2011-2012. IEPC-ACG-027/12

\section{Considerando}

\section{(...)}

"El modelo o sistema electrónico para la recepción del voto que apruebe el Conejo (sic) General de este organismo electoral, garantizará el respeto y apego a los principios rectores de la función electoral para la emisión del voto" (...)

\section{Acuerdo}

"Se aprueban los lineamientos para el uso del sistema electrónico para la recepción del voto mediante urna electrónica, durante el proceso electoral ordinario 2011-2012" (...)

LINEAMIENTOS PARA EL USO DEL SISTEMA ELECTRÓNICO PARA LA RECEPCIÓN DEL VOTO MEDIANTE URNA ELECTRÓNICA, EN EL PROCESO ELECTORAL ORDINARIO 2011-2012

\section{DOCUMENTACIÓN Y MATERIAL ELECTORAL}

(...)

"Los modelos de boleta electrónica aprobados deberán contener los siguientes elementos:

Entidad, distrito electoral uninominal y municipio

Elección de que se trate

Emblema, color o colores de cada partido político que haya registrado candidatos para la elección que corresponda

Nombre (s) y apellidos del candidato o de los candidatos

Emblema del Instituto

Las firmas del Consejero Presidente y Secretario Ejecutivo del Consejo General” (...) 
"El presidente de casilla ubicará la urna electrónica en un lugar visible, cuidando que en todo momento se garantice la secrecía del voto, su resguardo de las inclemencias del clima, y mostrando a los representantes de partido que el contenedor de testigos de voto se encuentra vacío" (...)

\section{DESARROLLO DE LA VOTACIÓN}

"Una vez concluido el proceso de "inicialización”, y se encuentre lista la urna electrónica con la aplicación abierta, el presidente, previa comprobación de los datos del ciudadano en el listado nominal, exhibición de su credencial para votar o en su caso, la resolución del Tribunal Electoral que les otorga el derecho a votar sin aparecer en la lista nominal o sin contar con credencial para votar o en ambos casos, permitirá el inicio de la votación accionando el código de "acceso al voto" por cada elector que se presente" (...)

"La urna electrónica contará con dos pantallas una táctil en la parte frontal para que el elector emita su voto y otra en la parte posterior para indicar el estado en el que se encuentra la urna" (...)

"La urna electrónica, una vez que el elector concluya el proceso de votación, guardará de manera aleatoria en una base de datos acumulativa, el sentido del voto realizado e imprimirá un testigo de voto" (...) 